\title{
Una bioetica per la persona: il caso del trapianto samaritano
}

\section{Mario Timio}

Dipartimento di Medicina Interna e Dialisi, Ospedale Foligno, Foligno (PG) Centro di Bioetica della Regione Umbria, Perugia

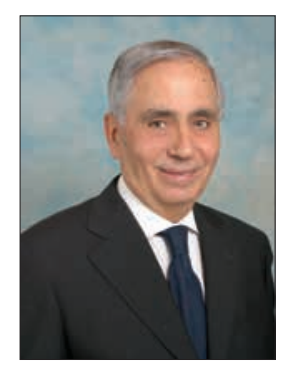

Mario Timio

\section{New ethical and legal issues in samaritan and cross-over transplantations}

One of the tragedies of modern medicine is that physicians now have the capability to save lives and prevent suffering but sometimes they are lacking in this capability. Organ, namely kidney, transplantation is one of this medical areas. But living donors are overcoming the problem. Samaritan and cross-over transplantations, as parts of nonrelated living donations, are a very promising approach to enhance living donation and to open a new era weaving the future. Samaritan donors are people who want to give an organ blindly to everyone regardless of sex, age, race and social status. But this donation creates some incoming ethic problems. A failed kidney transplantation from father to his son and a commercialized organ transfer are reported. Samaritan italian law and its bioethical implications are the most dramatic solidaristic intervention to prolong life as a cultural challenge of $21^{\text {th }}$ century.

Keywords: Samaritan transplantation, Bioethics in living donors (1). Il trapianto samaritano, come variante del trapianto da vivente, è stato normato dal Ministro della Salute Ferruccio Fazio nel 2010, su parere del Consiglio Superiore della Sanità e del Comitato Nazionale di Bioetica (CNB). Il ricorso al parere del CNB sottolinea la complessità bioetica che il trapianto sottende. A cominciare dal trapianto renale da vivente consanguineo o affettivamente legato. La descrizione di alcuni casi aiuta a entrare nella vasta complessità bioetica del trapianto da vivente e da samaritano.

\section{Accepted: June 25, 2015}

Published online: July 20, 2015

Indirizzo per la corrispondenza:

Prof. Mario Timio

Via XX Settembre 22

06121 Perugia

timma@libero.it

\section{Un trapianto d'amore finito male}

\section{Evento n. 1}

G.L., anni 31, ingegnere, figlio di un Professore universitario, titolare della cattedra di Pediatria. Da anni soffre di una forma di glomerulonefrite complicata da ipertensione arteriosa che, in breve tempo, vira verso l'insufficienza renale. Dietro I'angolo c'è la terapia sostitutiva: dialisi o trapianto renale da vivente. Prima del figlio, sceglie il padre. Senza esitare, offre il suo rene all'ingegnere che riceve, cosi, dal padre la vita due volte. Testata la compatibilità, viene effettuato il trapianto d'organo. La clinica va bene inizialmente per entrambi. Poi, I'incanto genitoriale inizia a incrinarsi. Il rene trapiantato mostra iniziali segni e sintomi di rigetto. L'aggravarsi del quadro clinico conduce l'ingegnere in emodialisi. Contestualmente, il padre avverte ematuria. Dopo ricoveri ed esami laboratoristici, la diagnosi che emerge è sconvolgente: carcinoma renale. Dopo un tentativo con la chemioterapia, la tappa terapeutica è solo la nefrectomia. Quindi, in emodialisi. Nello stesso ospedale, nello stesso reparto, nella medesima sala dialisi. Per scelta nello stesso turno, I'uno vicino all'altro. Anche per poter dialogare, per trovare o ritrovare quel pathos spesso assente tra padre e figlio, specialmente dopo una certa età. Ma il dialogo è durato poco, poiché il Professore un giorno, durante la seduta di emodialisi, pur nella sua carica di amore e di altruismo interpretati inutili, improvvisamente ha la sconcertante idea di morire. Gli era vicino il figlio, ancora in emodialisi. Non 
gli ha potuto stringere neanche la mano, poiché la fistola A.V. era costruita in entrambi a sinistra.

Certo, sembra un capitolo deamicisiano, ma un qualcosa che assomigli alla bioetica bisogna trovarlo. L'amore di un padre che dona un organo a un figlio non fa più notizia. Forse fa pensare che il padre abbia anticipato il senso delle parole espresse recentemente in una preghiera ebrea da Sheril Sandberg, CEO di Facebook, dopo la morte del marito: "Non lasciare che io muoia mentre sono ancora viva". E commenta così la sua stessa invocazione: "Solo adesso ho capito il significato che il tempo ha nella vita di una persona. Un valore incommensurabile quando si è ancora in vita". Proprio a questo valore faceva riferimento quel padre, che voleva dare più tempo alla vita del figlio della metà dei suoi anni. Nella guerra che la medicina sta vincendo in alcune nazioni, c'è la prospettiva che alcuni anni di vita "da vivi" possono fare la differenza. E il Professore lo sapeva che vivere "da vivo", pur con la malattia incombente, per il figlio significava nuove opportunità, nuove vite. Ma i suoi disegni sono andati in senso opposto. E la bioetica sta lì a indicare che tutto è stato inutile, investimenti falliti, prospettive annullate, desideri stracciati. Eppure sono state le regole essenziali della bioetica a far intraprendere quel percorso andato male. Trapianto fallito, inizio della dialisi, cancro del rene superstite (evento, peraltro, raro), morte. Peggio di così! A tanto non sarebbe giunto neanche Giobbe. Eppure, anche se la bioetica registra eventi tutti negativi, insegna che l'amore per l'altro e, segnatamente, per il figlio non è stato vano. Chiunque può raccogliere l'insegnamento e farne un veicolo di crescita umana e spirituale. La bioetica insegna che non è possibile negare anni di vita a chi può vivere decentemente e, addirittura, brillantemente, come l'ingegnere di cui sopra. Che può vivere totalmente, nella ricchezza di un'esistenza piena di affetti e di luoghi, di persone, di volti, di gusti e di ricordi, come del sacrificio di quel padre per il bene del figlio.

\section{Quando si vende il rene per miseria}

Una maggiore carica bioetica può emergere dalle peripezie sanitarie di un altro tipo di trapianto realizzato dalla vendita di un rene. Sul traffico illecito di organi la letteratura, non solo quella medica, è esuberantemente ricca. Si sa che il mercato e il turismo trapiantologici sono fiorenti in alcuni Paesi emergenti, come I'India, il Nepal, il Bangladesh e il Pakistan, a favore di altri sviluppati. Secondo Stephan Hughes, ordinario di antropologia all'Università di Berkeley, negli slum di Bombay, Calcutta, Karachi e Cairo, vegeta il commercio illegale di organi. È un bazar dove con 2000-3000 euro si compra ciò che si vuole, abitualmente un rene. A tal proposito, è da considerare la posizione della Transplantation Society che, nella sua dichiarazione di Istanbul (30 Aprile 2008), stabilisce di "prendere misure per proteggere i gruppi più poveri e vulnerabili del turismo trapiantologico e del mercato di tessuti e organi, con particolare attenzione al più vasto problema del traffico internazionale" (2). Malgrado questa dichiarazione, il traffico di organi persiste, per esempio al confine tra India e Pakistan, teatro della vicenda che caratterizza il prossimo evento.

\section{Evento n. 2}

F.H., anni 20, pakistano, abitante al confine con I'India, dalla quale è separato da un fiume. Al di là c'è una clinica privata per ricchi da trapiantare con organi di persone povere provenienti dall'altra sponda. Feisal, così lo chiameremo, è una di queste persone. Orfano di padre, madre malata, sei fratelli indigenti. Decide di attraversare il fiume. Si sottopone all'espianto, ritorna a casa con 3000 euro in più e un rene in meno. Che, in breve tempo, va incontro a disfunzione. II medico collegato con la clinica indiana, al quale si rivolge per oliguria, gli comunica, dopo una breve inefficace terapia, di recarsi all'estero per risolvere il suo problema. L'Italia sembra essere la meta più promettente. Con i pochi euro residui inizia un allucinante viaggio verso la salute sperata. Sbarca ad Ancona. Giunge alla mia osservazione presentato da un albanese in emodialisi. Terapia che inizia subito. Dopo alcuni mesi, gli chiedo come pensa di organizzarsi in Pakistan per continuare il trattamento dialitico. "Ho contattato uno dei numerosi centri dialisi diffusi nel mio Paese, a Peshawar, gestiti dalla fondazione Edhi. Se l'avessi conosciuto prima!". Ora Feisal è in attesa di un rene da trapiantare. Senza pagare, solo ricevere non denaro, ma vita.

In questo evento, la bioetica è interessata d'imperio. Intanto, non è abituale, in Italia, essere chiamati a risolvere problemi come quelli di Feisal. Si affrontano condizioni opposte, cioè di italiani che fanno fatto lo stesso percorso a ritroso per ricevere un rene che, nel tempo, non ha funzionato. Tutti in partenza per/ da bazar di organi: c'è chi vende, c'è chi compra. II commercio di organi illegali è diventato un business redditizio per numerose associazioni che, attraverso il web, riescono a lucrare sulle sofferenze dei malati e sulla miseria dei poveri (3). Abitualmente, un organo si dona per solidarietà, ma Feisal I'ha venduto per necessità. L'atto sembra travolgere ogni regola di umanità e dignità che pervade la medicina dai tempi di Ippocrate. Sembra che ogni traccia di etica e bioetica sia calpestata in questo commercio di organi umani, sottolineando che nessuno può creare forme di etica medica in astratto, ma è possibile solo estrarla dai fatti (4).

\section{Il nuovo sentiero della donazione samaritana}

Ma altri fatti conducono nel sentiero opposto a quello tracciato da Feisal nella e dalla clinica trapiantologica indiana. Già l'atto di donazione libera d'organo è un'alta e sublime forma di solidarietà umana. Ma la donazione samaritana è qualcosa di più. Il parere espresso dal CNB il 23 Aprile del 2010 ritiene che "I'atto di donazione samaritano è supererogativo e, quindi, eticamente apprezzabile. Deve avere, data la sua specificità, un carattere residuale e non sostitutivo (purché non esistano 
priorità biologiche di compatibilità) al trapianto da donatore cadavere o da donatore vivente consanguineo o affettivamente legato. II protocollo di donazione samaritana deve tenere conto di inserire prioritariamente il donatore samaritano nel programma di trapianti con modalità cross-over, definita come l'evento in cui il donatore e il ricevente non sono compatibili per la presenza di anticorpi anti-HLA o anti-ABO, ed è preclusa la procedura standard di trapianto da donatore vivente. In tal caso e in presenza di almeno un'altra coppia in una situazione analoga, i donatori e i riceventi, se biologicamente compatibili, si incrociano". Ho letto questo stralcio delle norme legislative sulla donazione samaritana a una persona che, dopo la pubblicazione delle medesime, ha ribadito di voler donare un rene. "Non chiedo nulla, non pongo condizioni, non mi interessa sapere se il ricevente sarà un uomo o una donna, sposato o meno, giovane o anziano, ricco o povero: chiedo solo di donare un organo per salvare una vita".

Quando ha saputo della prima donazione samaritana in Italia, si è rammaricato di non essere stato lui il primo anello della catena di trapianti incrociati di rene da vivente in modalità cross-over (5). Ma il potenziale samaritano ignora che, al di là del suo eventuale atto di grande umanità e solidarietà, esiste una serie di controlli ai quali deve sottoporsi anche per escludere falsi sentimenti umanitari. Come mai alcuni reclusi hanno fatto richiesta di donare un rene con la formula samaritana? Quindi, ben vengano norme bioetiche atte a preservare donatori da improvvise e improvvide iniziative, in una tematica che necessita di paletti volti a impedire che "mistici" temporanei trasporti altruistici si trasformino in postume richieste "monetizzanti". Proprio per capire le vere motivazioni di una donazione samaritana, oltre ai controlli medici specialistici, il Ministero della Salute ha predisposto visite psichiatriche e psicologiche che mettano al riparo da possibili falsi sentimenti di solidarietà. Al di là di ogni confusione procedurale, quello che conta è avere introdotto anche in Italia un principio di non secondaria importanza in campo trapiantologico: la donazione samaritana.

\section{Conclusioni}

Il trapianto samaritano, variante di quello da vivente, entrato recentemente nella legislazione italiana, costituisce un valido apporto per ridimensionare la cronica carenza di reni da trapiantare. Anche da vivente. La sua validità bioetica, stabilita anche dal Comitato Nazionale di Bioetica, impone, però, dei precisi limiti da rispettare, che non sono solo quelli biologici, ma che sono soprattutto di natura psichiatrica e psicologica, che mettano al riparo da possibili falsi sentimenti di umanitarismo, solidarietà e altruismo. Ciò perché, nella parola "samaritano", che sottolinea la misericordia di un uomo verso un altro uomo sofferente, si può indovare un "mistico" temporaneo trasporto altruistico, di cui, nel tempo, ci si può pentire e, contestualmente, si può verificare una possibile futura non gratuita richiesta "monetizzante". Bene, allora ha agito il Ministero della Salute, non solo ponendo dei precisi paletti psicologici, ma prevedendo che tutte le donazioni samaritane vengano trascritte in un Registro Nazionale e che, dopo te prime dieci donazioni, si faccia il punto della situazione dei donatori e dei riceventi. Se tutto va bene per tutti, si procede di dieci in dieci. Ciò per evitare confusioni estrumentalizzazioni e per sottolineare che la donazione samaritana non può essere una tecnica routinaria, ma solo eccezionale. Nel rispetto di precise norme di bioetica per la persona sana e malata.

\section{Disclosures}

Financial support: No financial support was received for this submission.

Conflict of interest: The author has no conflict of interest.

\section{Bibliografia}

1. Timio M. La sfida etica del trapianto. In: Timio M. Stringi la mia mano. Edizioni Porziuncola. Assisi 2011;43-8.

2. Recommendation on living organ transplantation. Nephrol Dial Transpl 2008;23:3375-80.

3. Bernabeu M. Meeting the challenge of transplantation in the 21th century. In: Kahan BD, Ponticelli C. New dimension in transplantation. Elsevier, New York 1988;30:1619-24.

4. Engelhardt HT. The rules of bioethics: moral conduct of health in a secular, morally pluristic context. In: Kjellstrand CM, Dossetor JB. Ethical problems in dialysis and transplantation. Kluwer Academic Publisher, Dordrecht 1992;3-19.

5. Jurez F, Barrios $Y$, Cano E, et al. Domino (Crossover) kidney transplantation using low doses of Neural. In: Kahan BP, Ponticelli C. Op. cit. 2289-90. 